\title{
Growth mechanism of periodic nanopattern in metal-oxide composites
}

\author{
Yu Wen ${ }^{1}$, Hideki Abe $^{2}$ and Ayako Hashimoto ${ }^{3}$
}

${ }^{1}$ University of Tsukuba, Tsukuba, Ibaraki, Japan, ${ }^{2}$ National Institute for Materials Science, United States,

${ }^{3}$ National Institute for Materials Science, Tsukuba, Japan

Noble metal-metal oxide composites (NM-MOs) at nanoscale are of interest because of improved catalytic activity by high surface-to-volume ratio [1]. Because of the restricted concentration of noble metals prepared via chemical routes, oxidation of binary alloy has been widely used to synthesize high surface NMMOs [2-3]. A variety of NM-MOs structures can be fabricated depending on the alloy's element type, composition, particle size, etc. An oxide scale with rich noble metal in the inside alloy has been obtained in $\mathrm{Pt}-\mathrm{Ni}$ alloy [4]. For $\mathrm{Au}-\mathrm{Sn}$ alloy nanoparticles, core-shell structures are formed due to the predominant diffusion of oxygen anions through the growing oxides [5]. Recently, lamellae and maze structures of Pt and $\mathrm{CeO} 2$ nanophases were observed in the composites prepared by annealing the Pt5Ce alloy [6]; the ionic conductivities of the composites were reported to be strongly sensitive to their structure. Therefore, understanding the growth mechanism of $\mathrm{Pt} \# \mathrm{CeO} 2$ composites is crucial for controlling the structure and corresponding ionic conductivity. In this work, the oxidation process in the Pt5Ce alloy was studied via highresolution TEM and STEM characterization.

Pristine Pt5Ce powders with an average size of approximately $50 \mu \mathrm{m}$ were synthesized by using the arcmelting method followed by grinding. Focused ion beam (FIB) was used to thin the powders for TEM observations. A double Cs-corrected TEM (JEM-ARM200F, JEOL) operated at $200 \mathrm{kV}$ was used to characterize the morphology and atomic structure of the materials. For the ex-situ experiment, an FIB-thinned specimen of the Pt5Ce alloy was annealed at $400{ }^{\circ} \mathrm{C}$ in $\mathrm{CO} 2$ and $\mathrm{O} 2$ syngas for $10 \mathrm{~min}$. A single crystal with an observation direction of [0001] was selected for the FIB-thinned specimen. After the annealing treatment, areas with different oxidation degrees were analyzed to understand the oxidation process. For verifying the results obtained from the ex-situ experiments performed on the FIB-thinned specimen, Pt5Ce powder samples annealed at $400{ }^{\circ} \mathrm{C}$ for $30 \mathrm{~min}$ and $12 \mathrm{~h}$ were thinned via FIB for TEM characterization.

Figure 1 shows the morphology and atomic structure of the pristine Pt5Ce alloy along the [0001] zone axis. A pure Pt5Ce alloy with numerous dislocations can be observed due to the fast cooling after arc-melting. Once the FIB-thinned specimen was oxidized for $10 \mathrm{~min}$ at $400^{\circ} \mathrm{C}$, a stripe pattern for the $\mathrm{Pt \# CeO} 2$ composite was formed with a periodicity of about $5 \mathrm{~nm}$, as shown in Fig.2. The black dashed line indicates the oxidation interface between the Pt5Ce alloy and $\mathrm{Pt} \# \mathrm{CeO} 2$ composites. It can be deduced that the oxidation proceeds by the predominant diffusion of oxygen anions through the $\mathrm{CeO} 2$ phase as well as by the opposite diffusion of metals $(\mathrm{Pt}, \mathrm{Ce})$. The high resolution HAADF-STEM image of the composites near the oxidation interface in Fig.2(b) shows an epitaxial relationship between $\mathrm{Pt}$ and $\mathrm{CeO}$. The growth direction of the oxide is along the [111] of $\mathrm{Pt \# CeO}$ 2. In addition, several specific orientation relationships between the alloy and composites were found at the oxidation interface. The oxidation products far away from the oxidation interface, which correspond to the final oxidation stage, were also analyzed. The results show that the epitaxial relationship between $\mathrm{Pt}$ and $\mathrm{CeO} 2$ breaks as a consequence of the crystals rotation. Such rotation behavior resulting in an incoherent interface has been reported at the heterointerface of NM-MOs because of the lower interface energy [7]. The power samples annealed for $30 \mathrm{~min}$ and $12 \mathrm{~h}$ represent the initial and final oxidation stages of the alloy, respectively. The results also show that an epitaxial relationship exists between $\mathrm{Pt}$ and $\mathrm{CeO} 2$ in the composites at the initial stage and then disappears in the final stage, which is consistent with the results obtained from the FIB-thinned specimen. The difference can be explained by the discrepancy in the direction along which the oxidation proceeds and the orientation relationship between the alloy and composites. This can be deduced from the discrepancy in the oxygen flow rate through the surface of the FIB-thinned specimen and the inside of the powder sample. 

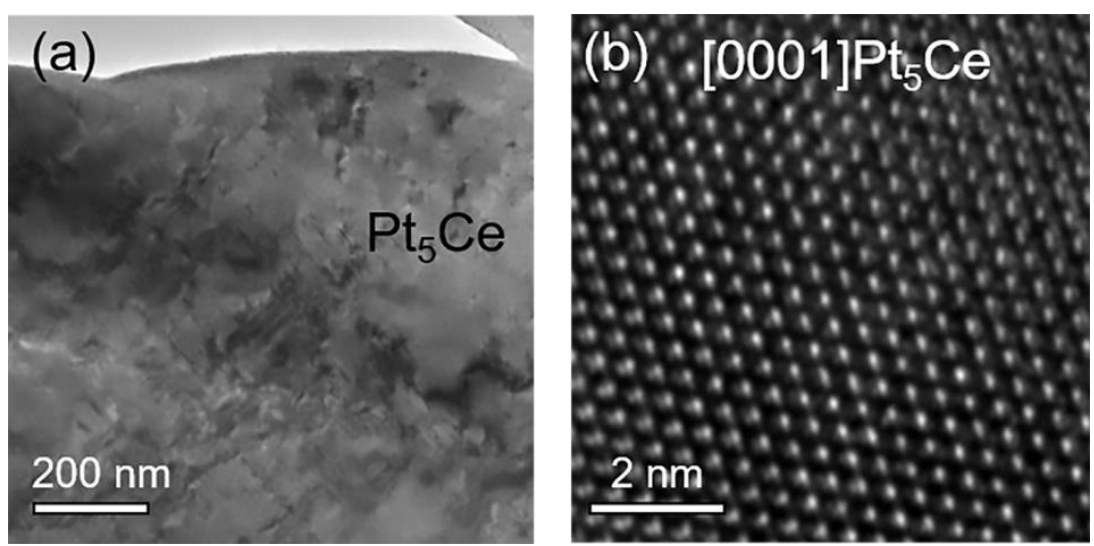

Figure 1. TEM (a) and HRTEM (b) images of Pt5Ce alloy along [0001] direction.
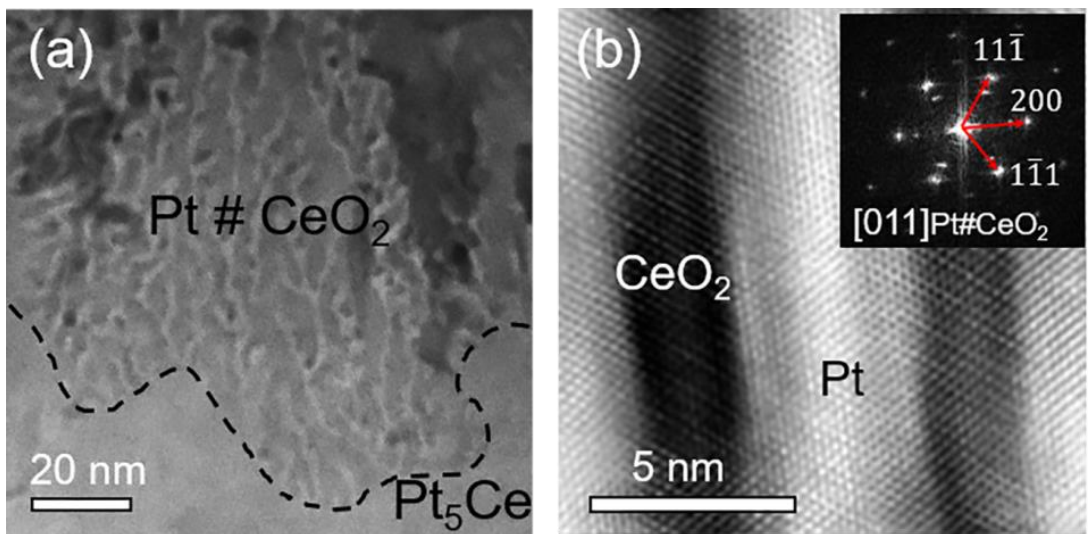

Figure 2. HAADF-STEM images of Pt5Ce alloy heated for $10 \mathrm{~min}$. (a) Pt\#CeO2 composites with lamellar structure was formed during the oxidation. (b) Epitaxially relationship between $\mathrm{Pt}$ and $\mathrm{CeO} 2$ was established from the high-resolution STEM image and corresponding FFT pattern.

\section{References}

[1] H. Goesmann and C. Feldmann, Angew. Chem., Int. Ed., 491362 (2010).

[2] E. A. Sutter et al., PNAS., 11010519 (2013).

[3] N. Sanz et al., Mater. Sci. Eng., C, 1979 (2002).

[4] C. Wagner, J. Electrochem. Soc., 99369 (1952).

[5] P. Sutter et al., Nanoscale Horiz., 1212 (2016).

[6] Y. Wen et al., Appl. Phys. Lett., 118054102 (2021)

[7] K. Matsunaga et al., Phys. Rev. B - Condens. Matter Mater. Phys., 742 (2006). 